\title{
Importance of specific rehabilitation methods for patients with intracerebral hemorrage
}

\author{
Ioana Stănescu',2, Gabriela Dogaru ${ }^{1,2}$, Rita Kallo², Adriana Bulboacă ${ }^{1,2}$
}

Corresponding author: Gabriela Dogaru, E-mail address: dogarugabrielaumf@gmail.com

\section{Abstract}

Intracerebral hemorrhage (ICH) is a severe type of stroke which causes bleeding in the brain parenchyma, accounting for 10-15\% of all strokes. Prognosis of patients with ICH remains very poor despite new advances in management strategies, and has not improved in the last decades. Disability remains important in ICH survivors, only a small proportion of patients live an independent life after ICH. Development of new strategies in rehabilitation care is expected to improve survival and outcome after intracerebral hemorrhage. The main neurological sequellae after ICH are similar to those seen after ischemic stroke. Given the clinical and pathological differences between the two types of stroke, clinical recovery is expected to be different. Comparisons between rehabilitation results in hemorrhagic versus ischemic stroke have offered mixed results, but all of them agreed that rehabilitation is highly effective after hemorrhagic strokes. There are no standard recommendations regarding the specific procedures used in the rehabilitation program of ICH patients. In the absence of clinical data to guide specific practice, the rehabilitation of ICH patients is largely based on general principles learned from ischemic stroke recovery. Physical therapy, task-specific training, sensory stimulation, use of assistive devices for upper limb or gait functions, speech and language therapy, physiotherapy and balnear therapy are methods used in rehabilitation of patients with hemorrhagic stroke, with improvement in patients functional outcome. There is a need for further development of specific rehabilitation techniques to enhance recovery in ICH patients.

Key words: intracerebral hemorrhage, hemorrhagic stroke, rehabilitation, functional outcome

\section{Introduction}

Intracerebral hemorrhage (ICH) is a type of stroke that occurs after rupture of a blood vessel within the brain parenchyma and accounts for $10-15 \%$ of all strokes [1, 2]. Primary intracerebral hemorrhage refers to the spontaneous rupture of small vessels damaged by chronic hypertension or amyloid angiopathy, in the absence of an underlying lesion, and is the most frequent type of intracerebral hemorrhage (78- 88\% of all ICH); in secondary intracerebral hemorrhage, bleeding occurs on a preexisting lesion, such as vascular malformation or tumour [2], or is associated with impaired coagulation (anticoagulant, antiplatelet or thrombolytic treatments). Intracerebral hemorrhage incidence was 24.6 per 100000 person-years, and was not significantly lower in women than in men [3]. The overall intracerebral hemorrhage mortality rates are about $40 \%$ at 1 month [2], 34\% at 3 months [4] and $54 \%$ at 1 year [3].

\section{Prognostic factors for outcome in acute ICH}

Prognosis of patients with ICH remains very poor despite new advances in management strategies, and has not improved in the last decades [2]. Development of new and more aggressive methods of medical and surgical management and of new sequences in rehabilitation care is expected to improve survival and outcome after intracerebral hemorrhage $[2,5]$.

Clinical predictors of poor outcome are: older age, pre-existent cognitive impairment, early decline in Glasgow Come Score (GCS), status of "do-notresuscitate" (DNR) orders, high systolic blood pressure (SBP), anticoagulant treatment, occurrence of seizures, fever, hyperglycemia, increase in Neutrophil / Lymphocyte ratio and high serum fibrinogen levels [6]. Radiographic predictors of poor outcome are: large hematoma volume, localization of hemorrhage in deep areas, hematoma expansion, presence of "spot sign" on contrast-enhanced computer-tromography (CT) scan, and "swirl sign" on native CT scan, important perihematomal edema, intraventricular hemorrhage and presence of hydrocephalus [6]. There are many factors that can change the survival rate and interfere with the degree of recovery in ICH patients.

Prognostic factors for outcome in post-acute ICH Disability remains important in ICH survivors, the proportion of patients living an independent life after intracerebral haemorrhage varies between $12 \%$ and $39 \%$ [1-3], imposing an important burden on healthcare and rehabilitation systems. Rehabilitation after hemorrhagic stroke has always been a challenge, considering specific physiopathologic particularities of $\mathrm{ICH}$, and the different impact of clinical and 
radiological predictors of ICH severity on disability status. Given the clinical and pathological differences between ischemic and hemorrhagic stroke, it is not surprising that clinical recovery also differ.

The patients with ICH had more severe clinical picture and greater functional impairment at admission than ischemic stroke patients. Despite greater mortality rate in early $\mathrm{ICH}$, many studies have shown a better functional recovery for ICH patients undergoing rehabilitation programs. Differences in functional outcome favoring patients with hemorrhagic stroke were described in different studies [7,8]. ICH patients showed a higher Barthel Index [7] and a greater change in Functional Independence Measure (FIM) total score at discharge from rehabilitation facility than ischemic stroke patients [8]. Patients with severe ICH showed more improvement than those with cerebral infarction of comparable severity; functional outcome after rehabilitation was predicted by initial degree of disability, age, and duration of therapy [8]. Contrary, there were other studies showing similar improvements of functional status at discharge in both types of stroke [9], or even poorer outcome and increased disability in ICH survivors [10]. Comparisons between rehabilitation results in ICH versus ischemic stroke have offered mixed results, but all of them agreed that rehabilitation is highly effective after hemorrhagic strokes [11].

Methods. The recommendations for rehabilitation treatment in hemorrhagic stroke are extensive regarding efficacy, duration and timing, but are sparse concerning the specific methods that need to be used in ICH patients. There are only few studies in the literature addressing the methods of rehabilitation which are efficient in hemorrhagic stroke.

Onset and duration of rehabilitation program in $\mathrm{ICH}$ were assessed in many studies. In patients with hemorrhagic stroke, early rehabilitation in the first month after stroke onset - average between 19 - 20 days - is showed to be effective, leading to better clinical outcomes in both the physical and mental health of the patients, and to better improvement of Activities of Daily Living (ADL) and motor function than in the group with late onset [12]. According to this study, the biggest improvement was achieved in the first month post- $\mathrm{ICH}$, and the rate of recovery gradually slows down and reaches a plateau by the end of six months [12] .
The main neurological sequellae after ICH are similar to those seen after ischemic stroke: motor and sensory deficits, aphasia, neglect, cognitive dysfunction or depression [13]. After completing rehabilitation program, different results were obtained in the recovery of upper and lower limb function, sensory function or trunk control. A Korean study of 11 patients with ICH, which completed 6 months of rehabilitation, showed that trunk control, lower limb motor function and sensory function improved significantly from the beginning and in the first 3 months, with no change after that interval. Upper limb function and gait remained not changed in the first month after stroke onset, but then showed significant improvement at 3 months, and continued to improve for 6 months [14].

Differences in motor recovery between ICH patients were also shown to be related to the location and extent of brain lesion. Patients with preserved integrity of the pyramidal tract showed better motor function than patients with discontinued pyramidal tract [15]. Involvement of the internal capsule, thalamus, periventricular white matter, and premotor cortex might be related to poor recovery of the upper limbs function in patients with hemorrhagic stroke [14]. The conclusions of these studies were that rehabilitation after ICH should start early (in the first 3 weeks) and continued for 6 months.

The duration of rehabilitation procedures depends on the setting where rehabilitation procedures are provided. In inpatient facilities, patients receive intense programs, 3 hours of daily therapy 5 to 7 days per week, but for shorter periods (usually less than 1 month). In outpatient facilities or in home-based recovery, the duration is $0.5-1$ hour per day of therapy, 2-3 times per week, but for 1 to 2 months [13].

\section{Rehabilitation procedures used in patients with intracerebral hemorrhage}

There are no standard recommendations regarding the specific procedures used in the rehabilitation program of ICH patients. In the absence of clinical data to guide specific practice, the rehabilitation of ICH patients is largely based on general principles learned from ischemic stroke recovery. Also, neurological deficits addressed by rehabilitation techniques are similar in hemorrhagic stroke and ischemic stroke, assuming that the use of same methods is effective.

Comprehensive treatment plans are specifically tailored to each patient, depending on the nature and degree of neurological impairment. 
Methods used for rehabilitation of ICH patients differ among centers. Classical rehabilitation procedures are based on physical therapy (neurodevelopmental approach) and occupational therapy (task oriented approach).

\section{Physical therapy in ICH}

Physical therapy interventions in acute stages of ICH and in patients with severe motor deficit are based on passive mobilization (passive musculoskeletal intervention): range of motion, positioning (to promote joint anatomic alignment), transfer techniques [16]. In subacute stages of ICH, active musculoskeletal interventions are initiated, focused on using and strengthening the affected limb, on symmetric weight bearing and transfer activities, and on gait training or ambulation with assistive devices. In ICH patients with lower extremity sensorimotor impairment associated with neglect or ataxia, physical therapy procedures specifically involve bed mobility and transfers training, gait exercises, balance, lower extremity range of motion and strength, and stair navigation. In ICH patients with upper extremity sensorimotor impairment, associated with apraxia or ataxia, physical therapy will mainly focus on optimizing ADL independence [13].

\section{Occupational therapy in ICH}

Task-oriented training is emerging as the dominant and most effective approach to motor rehabilitation of upper extremity function after stroke [17], this technique implies organization of the movement around a behavioral goal that is constrained by the environment [18]. In the literature there are no recommendations for task-specific training for patients with hemorrhagic stroke. Functional task training involves a task oriented approach in many ADL domains, such as: training of independent eating, bathing or dressing, training of transfer and locomotion [19]. Adaptive equipment (e.g., built-up utensils, walkers, tub benches, grab bars and rails) are useful also in ICH patients to improve safety and functionality at home.

A recent study shows no evidence of a dose-response effect of task-specific training on functional capacity in people with long-standing upper limb paresis post stroke [20].

\section{Assistive device or modality in patients with hemorrhagic strokes}

Patients with motor deficits after stroke may benefit of many assistive devices aimed to improve their upper limb or lower limb motor functions. For lower limbs motor deficit and gait training, techniques include body weight-supported treadmill training (BWSTT) and automated locomotion therapy with robotic devices: robotic-assisted gait training (RAGT). After treatment sessions, both hemorrhagic and ischemic stroke subjects showed significant improvements in lower limb and gait function [21].

Robot-aided therapy in the active-assisted mode offer highly repetitive, reproducible, interactive forms of training for the paretic upper limb, allowing easy and objective assessment of motor performance of the patient by recording biomechanical data [22]. Robotic devices significantly improve motor function in patients with long-term upper-limb deficits after stroke.

\section{Speech and language therapy in ICH}

Speech and language pathologists address the dysphagia, dysarthria, aphasia, and cognitive impairments encountered in the ICH patient, the techniques being similar to those used in ischemic stroke [13]. Intensive speech and language therapy is beneficial even in patients with chronic aphasia after stroke, by significantly enhancing verbal communication (study performed in people aged less than 70 years) [23].

\section{Sensory stimulation in ICH}

Patients with sensitive impairment undergo sensory stimulation. The modulation of sensory afferents is then proposed, using different methods, such as repetitive cutaneous electrical stimulation (on the hand and fingers with sensory deficit) [24], constraint-induced movement therapy, mirror therapy and virtual reality training. All sensory stimulation techniques were described in both ischemic and hemorrhagic stroke patients.

Physiotherapy Physiotherapy appeared to be an effective method to promote motor recovery poststroke, yet the precise modalities of an effective therapy were not properly definened, neither specifical applications in rehabilitation of hemorrhagic strokes.

\section{ICH patients with co-morbidities:}

Patients with hemorrhagic stroke usually have one or more cardiovascular risk factors, such as hypertension - the most important risk factor, current smoking, excessive alcohol consumption, old age, male sex, chronic kidney disease [ 25], and multiple other comorbidities, like diabetes mellitus, atrial fibrillation, ischemic heart disease, alcoholic liver disease, chronic arterial occlusive disease [26]. Besides specific drug therapy for these conditions, 
rehabilitation treatment should address specific functional impairments caused by these conditions. For that reason, a multidisciplinary team is required, especially for assessing patient's limitations in performing specific tasks and for assisting patient's safety [27].

The choice of a facility who is able to perform multitask rehabilitation is a difficult choice: inpatient rehabilitation hospitals in acute and subacute phase, outpatient ("ambulatory") clinics in subacute phase and balnear resorts in chronic ICH patients.

\section{Improvement in quality of life of ICH patients: role of balnear therapy}

For patients with chronic neurological deficits after hemorrhagic stroke, an alternative to outpatient rehabilitation clinics are balnear resorts. In balnear resorts, the use of natural therapeutic factors (i.e. carbonated mineral water and bioclimate through aerotherapy) is combined with physical therapy and physiotherapy, and the result is a significant improvement not only in sensorimotor deficits and mental health, but also in the quality of life of stroke patients [28, 29].

Rehabilitation program of chronic ICH patients performed in balnear resorts allows also specific treatments of associated medical problems [30].

\section{Conclusions}

The current knowledge about ICH rehabilitation methods is under development. Intracerebral hemorrhage is a special type of stroke, with specific pathogenesis and evolution. Specific mechanisms are involved during recovery phase. There is a need to develop specific rehabilitation techniques to enhance these mechanisms in ICH. Optimal parameters for rehabilitation treatment (time of onset, intensity, duration) needs further assessments during clinical studies. There is hope that combining physical methods with bioengineering devices and with pharmacological treatment will further improve functional outcome of hemorrhagic stroke patients.

\section{Bibliography}

1. Qureshi AI, Tuhrim S, Broderick JP, Batjer HH, Hondo H, Hanley DF. Spontaneous intracerebral hemorrhage. N Engl J Med. 2001 May 10;344(19):1450-60

2. Ikram A, Wieberdink RG, Koudstaal PJ. International Epidemiology of Intracerebral Hemorrhage. Curr Atheroscler Rep. 2012 Aug; 14(4): 300-306
3. van Asch CJ, Luitse MJ, Rinkel GJ, van der Tweel I, Algra A, Klijn CJ. Incidence, case fatality, and functional outcome of intracerebral haemorrhage over time, according to age, sex, and ethnic origin: a systematic review and meta-analysis. Lancet Neurol. 2010 Feb;9(2):167-76.

4. Weimar C, Weber C, Wagner M, Busse O, Haberl RL, Lauterbach KW, Diener HC; German Stroke Data Bank Collaborators. Management patterns and health care use after intracerebral hemorrhage. a costof-illness study from a societal perspective in Germany. Cerebrovasc Dis. 2003;15(1-2):29-36.

5. Qureshi AI, Mendelow AD, Hanley DF. Intracerebral Hemorrhage. Lancet. 2009 May 9;373(9675):1632-44

6. Al-Mufti F, Thabet AM, Singh T, El-Ghanem M, Amuluru K, Gandhi CD. Clinical and Radiographic Predictors of Intracerebral Hemorrhage Outcome. Intervent Neurol 2018;7:118-136

7. Paolucci S, Antonucci G, Grasso MG, et al. Functional outcome of ischemic and hemorrhagic stroke patients after inpatient rehabilitation: a matched comparison. Stroke. 2003;34(12):28612865

8. Kelly PJ, Furie KL, Shafqat S, Rallis N, Chang Y, Stein J. Functional recovery following rehabilitation after hemorrhagic and ischemic stroke. Arch Phys Med Rehabil. 2003;84(7):968-972

9. Perna R, Temple J. Rehabilitation Outcomes : Ischemic versus Hemorrhagic Strokes. Behav Neurol. 2015;2015:891651

10. Barber M, Roditi G, Stott DJ, Langhorne P. Poor outcome in primary intracerebral haemorrhage: results of a matched comparison. Postgrad Med J. 2004 Feb;80(940):89-92

11. Kitago $\mathrm{T}$, Ratan RR. Rehabilitation following hemorrhagic stroke: building the case for strokesubtype specific recovery therapies. F1000Res. 2017;6:2044

12. Bai Y, Hu Y, Wu Y, Zhu Y, He Q, Jiang C, Sun L, Fan W. A prospective, randomized, single-blinded trial on the effect of early rehabilitation on daily activities and motor function of patients with hemorrhagic stroke. J Clin Neurosci. 2012 Oct;19(10):1376-9

13. Saulle MF, Schambra HM. Recovery and Rehabilitation after Intracerebral Hemorrhage. Semin Neurol. 2016 June ; 36(3): 306-312.

14. Lee KB, Kim JS, Hong BY, Kim YD, Hwang BY, Lim SH. The Motor Recovery Related with Brain Lesion in Patients with Intracranial Hemorrhage. Behav Neurol. 2015; 2015: 258161

15. Jang SH, Park JW, Choi BY, Kim SH, Chang CH, Jung YJ, Choi WH, Seo YS. Difference of recovery course of motor weakness according to state of 
corticospinal tract in putaminal hemorrhage. Neurosci Lett. 2017 Jul 13;653:163-167

16. Rand ML, Darbinian JA. Effect of an evidence-based mobility intervention on the level of function in acute intracerebral and subarachnoid hemorrhagic stroke patients on a neurointensive care unit. Arch Phys Med Rehabil. 2015 Jul;96(7):1191-9

17. Schweighofer N, Choi Y, Winstein C, Gordon J. Task-Oriented Rehabilitation Robotics. Am J Phys Med Rehabil. 2012 Nov;91(11 Suppl 3):S270-9

18. Byoung-Jin Jeon, Won-Ho Kim \& Eun-Young Park (2015) Effect of task-oriented training for people with stroke: a meta-analysis focused on repetitive or circuit training. Topics in Stroke Rehabilitation, 22:1, 34-43.

19. Petruševičienė D, Kriščiūnas A. Evaluation of activity and effectiveness of occupational therapy in stroke patients at the early stage of rehabilitation. Medicina (Kaunas) 2008; 44(3):216-224.

20. Lang CE, Strube MJ, Bland MD, et al. Dose-response of task-specific upper limb training in people at least 6 months post stroke: A Phase II, single-blind, randomized, controlled trial. Annals of neurology. 2016;80(3):342-354.

21. Dierick F, Dehas M, Isambert J-L, Injeyan S, Bouche A-F, Bleyenheuft $Y$, et al. Hemorrhagic versus ischemic stroke: Who can best benefit from blended conventional physiotherapy with robotic-assisted gait therapy? PLoS ONE; 2017: 12(6): e0178636

22. Mazzoleni S, Duret C, Grosmaire AG, Battini E. Combining Upper Limb Robotic Rehabilitation with Other Therapeutic Approaches after Stroke: Current Status, Rationale, and Challenges. BioMed Research International, vol. 2017, Article ID 8905637, 11 pages, 2017

23. Breitenstein C, Grewe $\mathrm{T}$, Flöel A, Ziegler $\mathrm{W}$, Springer L, Martus $\mathrm{P}$, Huber W, Willmes $\mathrm{K}$, Ringelstein EB, Haeusler KG, Abel S, Glindemann R, Domahs F, Regenbrecht F, Schlenck KJ, Thomas M, Obrig H, de Langen E, Rocker R, Wigbers F, Rühmkorf C, Hempen I, List J, Baumgaertner A; FCET2EC study group. Intensive speech and language therapy in patients with chronic aphasia after stroke: a randomised, open-label, blindedendpoint, controlled trial in a health-care setting. Lancet. 2017 Apr 15;389(10078):1528-1538.

24. Kattenstroth JC, Kalisch T, Peters S, Tegenthoff M, Dinse HR. Long-term sensory stimulation therapy improves hand function and restores cortical responsiveness in patients with chronic cerebral lesions. Three single case studies. Front Hum Neurosci. 2012; 6: 244.

25. An SJ, Kim TJ, Yoon BW. Epidemiology, Risk Factors, and Clinical Features of Intracerebral Hemorrhage: An Update. Journal of Stroke 2017;19(1):3-10.
26. Tseng CH, Muo CH, Hsu CY, Kao CH. Increased Risk of Intracerebral Hemorrhage Among Patients With Hepatitis C Virus Infection. Medicine: November 2015 - Volume 94 - Issue 46 - p e2132

27. Frîngu F, Guşetu G, Iosip A, Gurzău A, Dogaru G, Zdrenghea D, Pop D. The predictors of exercise capacity impairment in diabetic patients. Balneo Research Journal. 2017;8(2):26-32

28. Dogaru G, Ispas A, Bulboacă A, Motricală M, Stănescu, I. Influence of balnear therapy at Băile Tuşnad on quality of life of post-stroke patients. Balneo Research Journal. 2017;8(4):201-205

29. Dogaru G, Ispas A, Stănescu I, Motricala M, Molnár Á. A clinical study on the efficacy of natural therapeutic factors in Băile Tuşnad for the rehabilitation of post-stroke patients. Balneo Research Journal. 2017;8(1):5-10

30. Dogaru G, Stănescu I, Pop D, Motricală M, Molnár A. Effects of carbonated mineral water treatment in Băile Tuşnad on chronic arterial occlusive disease - a case report.Balneo Research Journal. 2017;8(3):121124 